\title{
MAXIMIZING HEMODYNAMIC EFFECTIVENESS OF BIVENTRICULAR ASSISTANCE BY DIRECT CARDIAC COMPRESSION STUDIED IN EX VIVO AND IN VIVO CANINE MODELS OF ACUTE HEART FAILURE
}

John H. Artrip, MD

Geng-Hua Yi, MD

Juichiro Shimizo, $\mathrm{MD}, \mathrm{PhD}^{\mathrm{b}}$

Eduard Feihn, MD

Robert R. Sciacca, EngScD ${ }^{\mathrm{b}}$

Jie Wang, MD, $\mathrm{PhD}^{\mathrm{b}}$

Daniel Burkhoff, $\mathrm{MD}, \mathrm{PhD}^{\mathrm{b}}$
Objective: Direct cardiac compression improves effective ventricular contractility. However, associated reductions in filling volumes and increases in arterial pressure occurring at the onset of direct cardiac compression limit the degree to which cardiac output is augmented. We tested the hypothesis that active preload and afterload control maximizes the hemodynamic effectiveness of direct cardiac compression.

Methods and results: Studies in isolated canine hearts loaded with a computer-controlled volume servo system that mimicked heart failure were used to clearly define the hemodynamic effects of direct cardiac compression. Immediately on initiation of direct cardiac compression, ventricular enddiastolic pressure and volume decreased substantially, arterial pressure increased, but stroke volume did not change significantly. When end-diastolic pressure was restored to about $20 \mathrm{~mm} \mathrm{Hg}$, stroke volume doubled; decreasing afterload resistance further increased stroke volume by about $30 \%$. Such load adjustments were then tested in vivo in a canine model of acute heart failure induced by coronary artery microembolizations titrated to decrease cardiac output to $33 \% \pm 9 \%$ of control as end-diastolic pressure rose to $20.6 \pm 2.2 \mathrm{~mm} \mathrm{Hg}$. Direct cardiac compression decreased end-diastolic pressure to $11.4 \pm 2.6 \mathrm{~mm} \mathrm{Hg}$ while increasing cardiac output from 0.8 \pm 0.2 to $1.4 \pm 0.5 \mathrm{~L} / \mathrm{min}$ (to only $\sim 55 \%$ of normal). Restoring end-diastolic pressure to $19.6 \pm 2.2 \mathrm{~mm} \mathrm{Hg}$ by infusions of saline solution increased cardiac output to $1.9 \pm 0.5 \mathrm{~L} / \mathrm{min}$. Afterload reduction (nitroprusside), while maintaining end-diastolic pressure at $19.8 \pm 1.3 \mathrm{~mm} \mathrm{Hg}$, increased cardiac output to its baseline, $2.8 \pm 1.1 \mathrm{~L} / \mathrm{min}$.

Conclusions: Direct cardiac compression significantly improves ventricular pumping capacity and can restore cardiac output to about $60 \%$ of normal in the setting of acute heart failure. When combined with active preload and afterload manipulations, direct cardiac compression can restore cardiac output to normal. (J Thorac Cardiovasc Surg 2000;120:379-86)
From the Department of Surgery, Division of Cardiothoracic Surgery, ${ }^{\mathrm{a}}$ and the Department of Medicine, Division of Circulatory Physiology, ${ }^{\mathrm{b}}$ College of Physicians and Surgeons, Columbia University, New York, NY.

Received for publication Aug 12, 1999; revisions requested Oct 20, 1999; revisions received March 1, 2000; accepted for publication March 6, 2000

This research was sponsored in part by an unrestricted grant from Cardio Technologies Inc, Pine Brook, NJ. Daniel Burkhoff and Jie Wang received grant support through Columbia University.

Address for reprints: Dan Burkhoff, MD, PhD, Department of Medicine, Division of Circulatory Physiology, College of Physicians and Surgeons, Columbia University, MHB 5-435, 177 Fort Washington Ave, New York, NY 10032 (E-mail: db59@columbia.edu).

Copyright (C) 2000 by The American Association for Thoracic Surgery

$0022-5223 / 2000 \$ 12.00+0 \quad \mathbf{1 2 / 1 / 1 0 6 9 8 6}$

doi: $10.1067 / \mathrm{mtc} .2000 .106986$
The use of synchronized biventricular epicardial compression achieved by inflation of a pneumatically driven bladder surrounding the heart has been proposed as a means of assisting the failing heart. ${ }^{1,2}$ Unlike other types of assist devices, such compression devices do not contact the blood and thus may be associated with fewer complications related to thromboembolic events. The physiologic principles by which direct cardiac compression (DCC) enhances ventricular pumping strength have been demonstrated in isolated canine hearts. ${ }^{3-6}$ The end-systolic pressurevolume relationship (ESPVR) is shifted upward (indicating increased effective contractile strength) by an amount proportional to the inflation pressure of the compression bladder. 


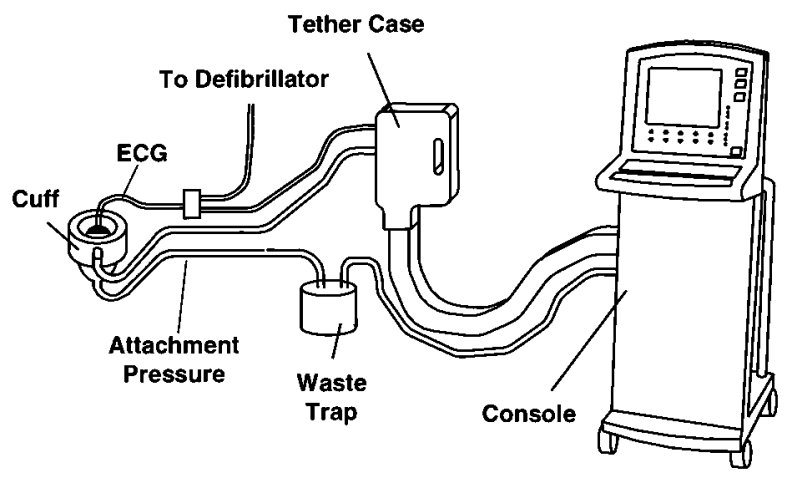

Fig 1. Schematic representation of the biventricular compression device (CardioSupport System [CSS], Cardio Technologies Inc, Pine Brook, NJ). ECG, Electrocardiogram.

In both the isolated ejecting heart and the heart in situ, application of biventricular DCC also results in decreased right ventricular (RV) and left ventricular (LV) preload volumes and pressures. ${ }^{6}$ On the basis of measurements showing that the end-diastolic pressure-volume relationship (EDPVR) is minimally altered by the device, ${ }^{7}$ it has been demonstrated that the reduction in preload is mainly a consequence of the increased pumping capacity of the supported heart. Although reductions of preload are clinically beneficial, they decrease the net flow and pressuregenerating capacity of the heart (Starling mechanism) even in the presence of DCC. ${ }^{6,7}$ For example, in a canine model of severe acute ischemic heart failure (defined as a reduction in cardiac output to $\sim 30 \%$ of normal), DCC doubled cardiac output, but net flow during support amounted to only about $60 \%$ of normal. This increase in cardiac output was associated with a decrease in LV end-diastolic pressure (EDP) from a mean value of 22 to $12 \mathrm{~mm} \mathrm{Hg}$ and an increase in arterial pressure from 55 to $100 \mathrm{~mm} \mathrm{Hg.}{ }^{7}$ However, according to physiologic principles, cardiac output can be normalized if preload and afterload are appropriately manipulated.

The purpose of this study was therefore to test whether manipulation of ventricular preload and afterload could enhance cardiac output in hearts with severe acute heart failure supported by DCC. An ex vivo isolated canine heart preparation, which allows precise measurement of ventricular volumes and control over preload and afterload, was used to elucidate the physiologic principles. The hemodynamic effects of DCC were then tested in vivo in a canine model of acute ischemic heart failure at different levels of compression pressures and during serial manipulations of preload and afterload.

\section{Methods}

A total of 20 adult male mongrel dogs (12 for isolated heart and 8 for acute in vivo studies) weighing between 22.0 and $27.6 \mathrm{~kg}$ (mean $\pm \mathrm{SD}, 24.1 \pm 1.8 \mathrm{~kg}$ ) were used for these studies. All animals received humane care in compliance with the "Guide for the Care and Use of Laboratory Animals" published by the National Institutes of Health (NIH Publication No. 85-23, revised 1985). For all studies, dogs were anesthetized with pentobarbital sodium $(30 \mathrm{mg} / \mathrm{kg}$, intravenously), intubated with an $8 \mathrm{~F}$ endotracheal tube, and maintained with mechanical ventilation using humidified room air (Harvard Apparatus Inc, Holliston, Mass).

DCC device. Mechanical biventricular DCC was provided by the CardioSupport System (CSS, Cardio Technologies Inc, Pine Brook, NJ). This device (Fig 1) consists of an inflatable cuff with 2 epicardial electrocardiogram sensors, a pneumatic pressure and vacuum generator, and a computer console. The cuff fits around the heart with an inflation bladder that circumscribes both ventricular chambers and covers approximately $50 \%$ of the epicardium. The cuff is held to the heart with negative attachment pressure $(\sim 200 \mathrm{~mm} \mathrm{Hg})$ applied to the LV apex. A solid-state MikroTip catheter (Millar Instruments Inc, Houston, Tex) placed in the driveline near the cuff is used to measure the pressure inside the inflation bladder. A digitally controlled mechanical pneumatic pump is used to control the amount $\left(\mathrm{P}_{\mathrm{DCC}}\right)$ and duration $\left(t_{D C C}\right)$ of compression pressure. The device can generate a $\mathrm{P}_{\text {DCC }}$ over $200 \mathrm{~mm} \mathrm{Hg}$ with $\mathrm{t}_{\mathrm{DCC}}$ varying between $10 \%$ and $50 \%$ of the cardiac cycle; $\mathrm{P}_{\mathrm{DCC}}$ values between 50 and 150 $\mathrm{mm} \mathrm{Hg}$ and $\mathrm{t}_{\mathrm{DCC}}$ values of $40 \%$ were typically used because prior studies have shown that these values provide optimal hemodynamic benefit. The electrocardiogram measured through the epicardial leads is used by the device to synchronize compressions to the intrinsic QRS complex.

Ex vivo canine heart study. Six isolated cross-perfused canine hearts (each experiment using 2 dogs) were studied by means of methods that were similar to those described previously. ${ }^{8}$ In brief, the femoral arteries and veins of 1 dog ("support dog") were cannulated and connected to a perfusion system used to supply oxygenated blood to the isolated heart. The heart from the second dog ("heart donor dog") was removed while metabolically supported by arterial flow from the support dog. Two metal adapters sutured to the mitral and tricuspid valve rings held the isolated heart to servo-pump systems used to measure and control the ventricular volumes via water-filled balloons placed inside each ventricle. A Millar pressure transducer placed inside each balloon was used to measure intracavitary pressures. The servo systems were controlled by a computer programmed to simulate the hydraulic impedances of the systemic and pulmonary circuits and allowed the ventricles to eject in series with each other in a physiologic manner. 9,10

Protocol. Heart rate was set between 100 and 140 beats/min by atrial pacing, and the values of the parameters of the simulated vascular systems were adjusted to approximate the hemodynamics of the heart failure state: RV EDP, 5 to $15 \mathrm{~mm} \mathrm{Hg}$; $\mathrm{LV} \mathrm{EDP,} 15$ to $20 \mathrm{~mm} \mathrm{Hg}$; stroke volume, 
Table I. The effect of increasing preload $(\uparrow P)$ and reducing afterload $(\downarrow A)$ on DCC-assisted ventricular function in isolated canine hearts under conditions of simulated heart failure

\begin{tabular}{|c|c|c|c|c|}
\hline & $H F$ & $H F+D C C$ & $D C C+\uparrow P$ & $D C C+\uparrow P+\downarrow A$ \\
\hline \multicolumn{5}{|l|}{ Left ventricle } \\
\hline ESP (mm Hg) & $91.7 \pm 6.1$ & $87.1 \pm 20.3$ & $115.6 \pm 13.9 * \dagger$ & $72.4 \pm 3.3^{*} \dagger$ \\
\hline $\mathrm{EDP}(\mathrm{mm} \mathrm{Hg})$ & $17.6 \pm 4.1$ & $8.0 \pm 2.2^{*} \dagger$ & $19.2 \pm 7.8 \dagger$ & $20.2 \pm 6.3$ \\
\hline ESV (mL) & $33.6 \pm 10.2$ & $15.9 \pm 6.4^{*} \dagger$ & $24.8 \pm 9.2^{* \dagger}$ & $12.9 \pm 3.2 * \dagger$ \\
\hline $\mathrm{EDV}(\mathrm{mL})$ & $38.7 \pm 10.2$ & $21.3 \pm 6.6^{* \dagger}$ & $34.3 \pm 6.7 \dagger$ & $24.9 \pm 5.9^{* \dagger}$ \\
\hline $\mathrm{SV}(\mathrm{mL})$ & $5.1 \pm 1.9$ & $5.7 \pm 1.7$ & $10.3 \pm 3.1 * \dagger$ & $12.7 \pm 4.4^{*}$ \\
\hline \multicolumn{5}{|l|}{ Right ventricle } \\
\hline ESP $(\mathrm{mm} \mathrm{Hg})$ & $37.9 \pm 10.9$ & $69.0 \pm 8.1^{* \dagger}$ & $67.7 \pm 8.7 *$ & $66.3 \pm 9.7 *$ \\
\hline $\mathrm{EDP}(\mathrm{mm} \mathrm{Hg})$ & $12.8 \pm 4.6$ & $8.9 \pm 5.7$ & $12.7 \pm 4.5$ & $11.7 \pm 5.7$ \\
\hline ESV (mL) & $22.2 \pm 8.4$ & $9.2 \pm 2.6^{* \dagger}$ & $8.3 \pm 6.3^{*}$ & $1.3 \pm 0.9 * \dagger$ \\
\hline $\mathrm{EDV}(\mathrm{mL})$ & $27.4 \pm 9.0$ & $15.1 \pm 4.4^{* \dagger}$ & $18.9 \pm 8.9$ & $13.5 \pm 7.8^{*}$ \\
\hline
\end{tabular}

about $5 \mathrm{~mL}$; peak arterial pressure, 90 to $100 \mathrm{~mm} \mathrm{Hg}$. Under these conditions, baseline ESPVR and EDPVR, as well as stroke volume-end-diastolic volume relations (EDV-SV) were obtained by recording pressure-volume data at 3 to 6 different preload volumes. Preload volume changes were obtained by simulating adjustments in the total circulatory volume of the computerized vascular system. The DCC device was then placed on the heart, compressions were initiated, and the measurements were repeated. Finally, the sequence was repeated after a reduction in simulated systemic vascular resistance.

In vivo canine heart study. A total of $8 \mathrm{dogs}$ were used to test the effects of adjusting $\mathrm{P}_{\mathrm{DCC}}$ and ventricular load on DCC-assisted ventricular function in vivo. For each in vivo experiment, the right carotid artery was cannulated with a Millar pressure transducer that was introduced into the left ventricle for measurement of LV pressure. The left carotid artery was cannulated with a Tygon catheter (Saint-Gobain Performance Plastics Corporation, Akron, Ohio) connected to a pressure transducer (Statham P32, Gould; Carolina Medical, King, NC) for measurement of arterial pressure. A lateral thoracotomy was performed through the fifth intercostal space. A flow probe (Transonic Systems Inc, Ithaca, $\mathrm{NY}$ ) was placed around the ascending thoracic aorta to measure cardiac output. A custom-made silicone catheter was introduced into the left anterior descending coronary artery that was used for coronary microembolizations to create heart failure. ${ }^{11,12}$

Coronary microembolization. Aliquots of $90 \mu \mathrm{m}$ glass beads $(\sim 25,000)$ were injected into the coronary artery every 3 to 5 minutes until a severe but stable level of acute heart failure was achieved ( $60 \%-70 \%$ of the normal baseline cardiac output). Ventricular ectopy was treated with intravenous lidocaine $(20 \mathrm{mg} / \mathrm{kg}$ loading dose followed by a constant infusion of $1 \mathrm{mg} \cdot \mathrm{kg}^{-1} \cdot \mathrm{min}^{-1}$ ).

Protocol. After the induction of stable heart failure and recording of hemodynamic data, an appropriately sized cuff was placed on the heart, and ventricular compressions were synchronized with the native electrocardiogram. $\mathrm{P}_{\mathrm{DCC}}$ was increased by increments of about $50 \mathrm{~mm} \mathrm{Hg}$ to obtain data at 50,100 , and $150 \mathrm{~mm} \mathrm{Hg}$. To test the effects of preload and afterload adjustments in vivo, we used normal saline solution to increase the EDP to about $20 \mathrm{~mm} \mathrm{Hg}$, followed by nitroprusside $\left(0.3-0.9 \mu \mathrm{g} \cdot \mathrm{kg}^{-1} \cdot \mathrm{min}^{-1}\right)$ to lower the mean arterial pressure to about $60 \mathrm{~mm} \mathrm{Hg}$. Volume loading was also repeated during nitroprusside infusion since the resulting venodilation decreased filling pressures. Data were recorded after each intervention and only after obtaining a stable state. Due to instability of this preparation, only 4 animals completed both the $\mathrm{P}_{\mathrm{DCC}}$ and ventricular loading portions of the protocol; thus, an additional 2 animals had to undergo only the ventricular loading segment of the protocol to achieve 6 successful experiments.

Data collection and statistical analysis. Data were recorded on an 8-channel chart recorder (Gould Instrument Systems Inc, Valley View, Ohio) equipped with an analog-to-digital converter; periods of interest were digitally sampled (1000 $\mathrm{Hz}$ ) and analyzed off-line.

For the isolated heart study, the end-systolic pressure (ESP) and volume (ESV) of each beat were determined by identifying the upper left hand corner of each pressure-volume loop and end-diastolic pressure (EDP) and volume (EDV) points were determined by identifying the bottom right hand corner. The ESPVRs and EDV-SV curves were characterized by linear regression analysis, whereas nonlinear regression was used for EDPVRs.

Statistical comparisons of the linear curves between control and test conditions were accomplished by repeated-measures analysis of covariance (RM-ANCOVA). Nonlinear EDPVRs were linearized (logarithmic transformation) before application of RM-ANCOVA. ${ }^{6,7}$ Hemodynamic parameters were expressed as mean $\pm \mathrm{SD}$ and were compared between various conditions by means of repeated-measures analysis of variance (RM-ANOVA) with indicator variables used to 


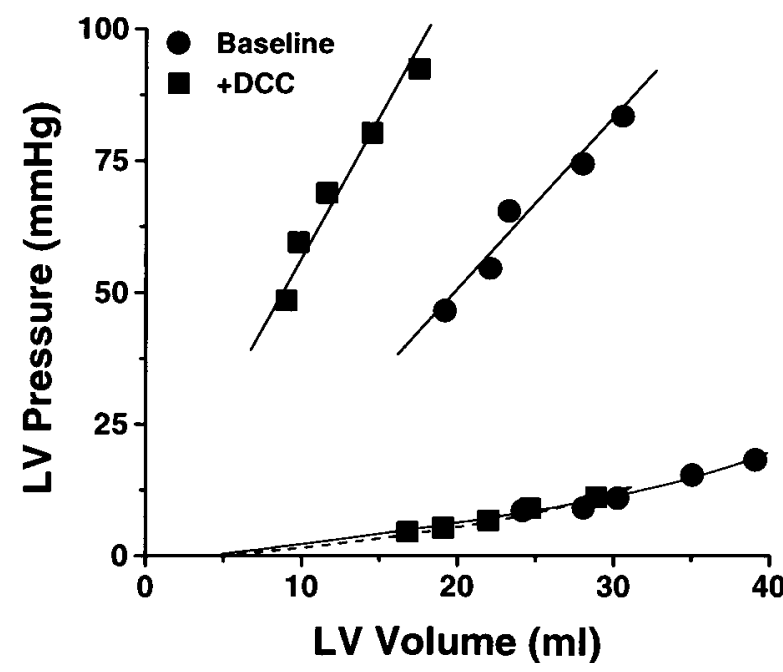

Fig 2. Effect of DCC on the LV ESPVR and EDPVR from a representative isolated canine heart. The ESPVR is shifted upward in response to DCC, whereas there is no statistically significant effect on the EDPVR. See text for details.

test the effects of specific hemodynamic interventions. ${ }^{13}$ Bonferroni corrections were used for post hoc tests to control for the effects of multiple contrasts. All statistical analyses were performed with commercially available software (SAS version 6.12, SAS Institute, Inc, Cary, NC).

\section{Results}

Effect of load adjustment on DCC-assisted hemodynamics in isolated hearts. Parameter values of the simulated vascular system were adjusted to mimic the hemodynamic conditions of heart failure as shown in Table I. Upon initiating DCC with a cuff pressure of about $100 \mathrm{~mm} \mathrm{Hg}$ (HF+DCC), RV and LV EDPs and EDVs decreased but stroke volume was not substantially affected. Volume loading was then simulated by increasing the simulated total intravascular volume so that RV and LV EDPs were increased to higher values $(\mathrm{DCC}+\uparrow \mathrm{P})$. This resulted in increases in ESPs, and stroke volume almost doubled from its baseline value. Systemic afterload resistance was then decreased (DCC+ $\uparrow \mathrm{P}+\downarrow \mathrm{A})$, which resulted in a decrease in $\mathrm{LV}$ ESP and a reduction in LV EDV with a further increase in stroke volume. These results show that initiation of DCC decreases preload volume and pressure and that hemodynamic effectiveness of DCC can be enhanced by manipulating preload and afterload.

To further clarify the mechanisms of DCC, we generated ESPVRs and EDPVRs from data obtained from these isolated hearts over a range of preload volumes. DCC shifted the ESP-ESV points toward lower ESVs and higher ESPs (Fig 2, data from a representative heart). On average, the slope of the ESPVR increased from 3.32 to $3.96 \mathrm{~mm} \mathrm{Hg} / \mathrm{mL}(P=.006$, RM-ANCOVA $)$ and the volume axis-intercept dropped from 1.9 to $-6.9 \mathrm{~mL}(P=$ $.03)$, both changes indicating an increase in effective ventricular contractility. DCC did not have a statistically significant effect on the EDPVR ( $P$ values from RMANCOVA for differences in slopes and elevations of the linearized EDPVRs were .55 and .74, respectively).

Data pertaining to the EDV-SV relation of these hearts at 2 levels of afterload resistance are shown in Fig 3 (representative example). At normal afterload, the average slope of EDV-SV relationship was unchanged by DCC ( 0.22 at baseline vs 0.20 during DCC, $P=.71$ by RM-ANCOVA); the y-axis intercept increased from -2.5 to $0.8 \mathrm{~mL}$ with DCC $(P=.06)$. With decreased afterload resistance, the slope increased from 0.3 to 0.4 $(P=.01)$; although the mean $\mathrm{y}$-axis intercept increased from -2.0 to $0.6 \mathrm{~mL}$, RM-ANCOVA did not reveal a statistically significant difference in mean elevation between these curves. RM-ANCOVA also revealed that although there was no significant difference between the baseline EDV-SV relations at the 2 different afterloads, there was a statistically significant difference in slopes during DCC between the 2 afterloading conditions $(P=.001)$.

Thus, for both levels of afterload, DCC shifted the EDV-SV relationship upward, indicating that at a given preload flow-generating capacity is increased significantly by DCC. These results reveal the physiologic mechanisms by which the effectiveness of the DCC can be maximized.

Effect of $\mathbf{P}_{\text {DCC }}$. DCC was next tested in the setting of acute ischemic heart failure in vivo $(n=6)$ at $P_{D C C}$ values of 50, 100, and $150 \mathrm{~mm} \mathrm{Hg}$ (Table II). Coronary embolization acutely decreased cardiac output, mean arterial pressure fell to $61.8 \pm 5.6 \mathrm{~mm} \mathrm{Hg}$, and EDP increased to $19.8 \pm 5.5 \mathrm{~mm} \mathrm{Hg}$. DCC-assisted cardiac output, stroke volume, mean arterial pressure, and LV systolic pressure increased progressively with increases in $\mathrm{P}_{\mathrm{DCC}}$, although only the changes in mean arterial pressure were statistically significant. In contrast, EDP incrementally decreased to $12.5 \pm 4.0 \mathrm{~mm} \mathrm{Hg}$. At the highest level of $\mathrm{P}_{\mathrm{DCC}}$, cardiac output was increased by about $50 \%$ compared with the unassisted heart failure state, but this still amounted to only about $50 \%$ of normal. Thus, as observed in the isolated hearts, the ability of DCC to increase cardiac output in vivo is hindered by decreases in filling pressure. For all parameters, there was only a marginal hemodynamic impact of increasing $\mathrm{P}_{\mathrm{DCC}}$ from 100 to $150 \mathrm{~mm} \mathrm{Hg}$. For this reason, further studies are performed at $\mathrm{P}_{\mathrm{DCC}}$ of $100 \mathrm{~mm} \mathrm{Hg}$. 

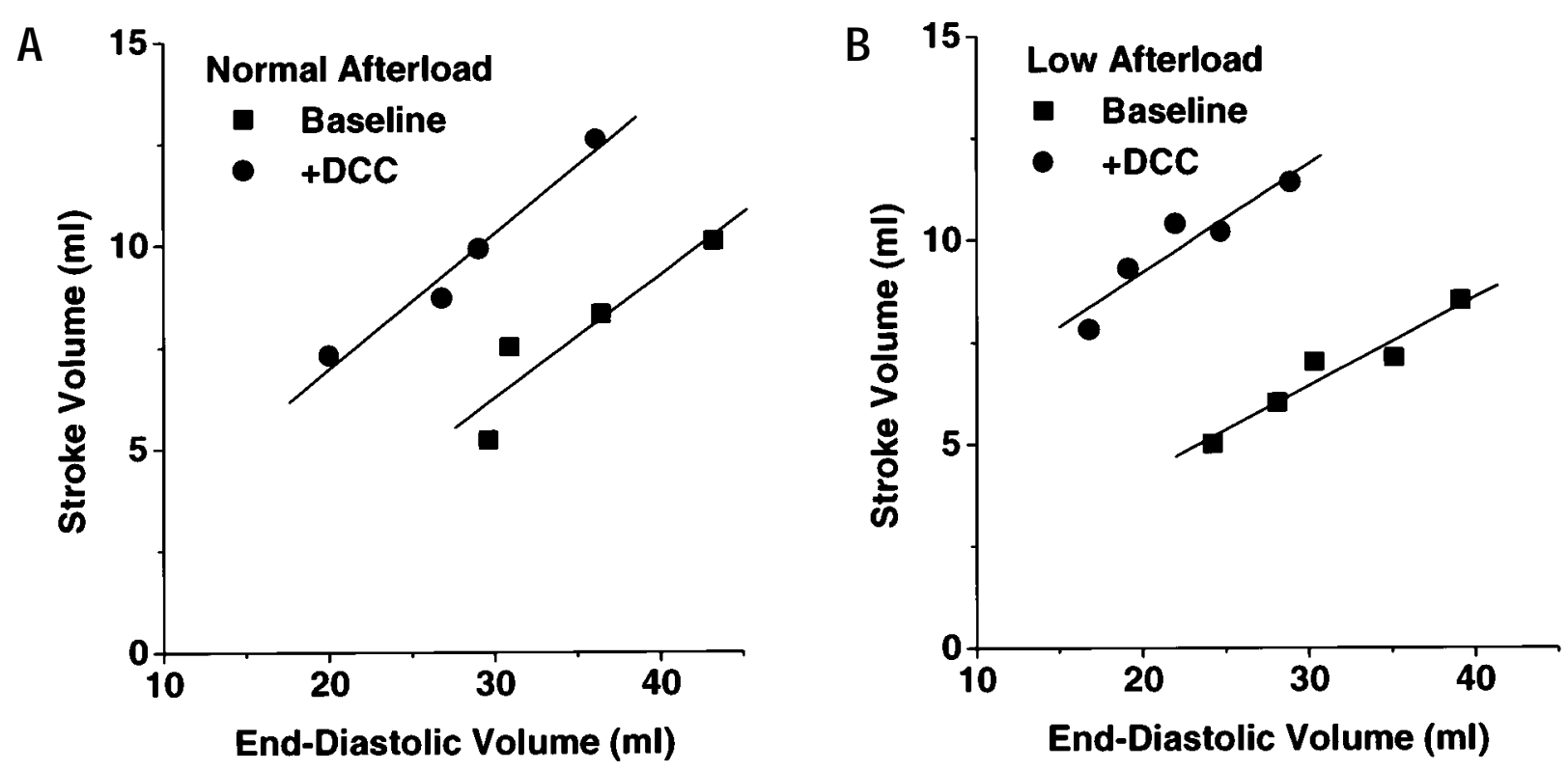

Fig 3. Effect of DCC on the relationship between end-diastolic volume and stroke volume at normal (A) and low (B) afterload arterial resistances from a representative heart. DCC shifts the end-diastolic volume-stroke volume relationship upward. See text for details.

Table II. The effect of compression pressure on hemodynamic parameters in ischemic heart failure

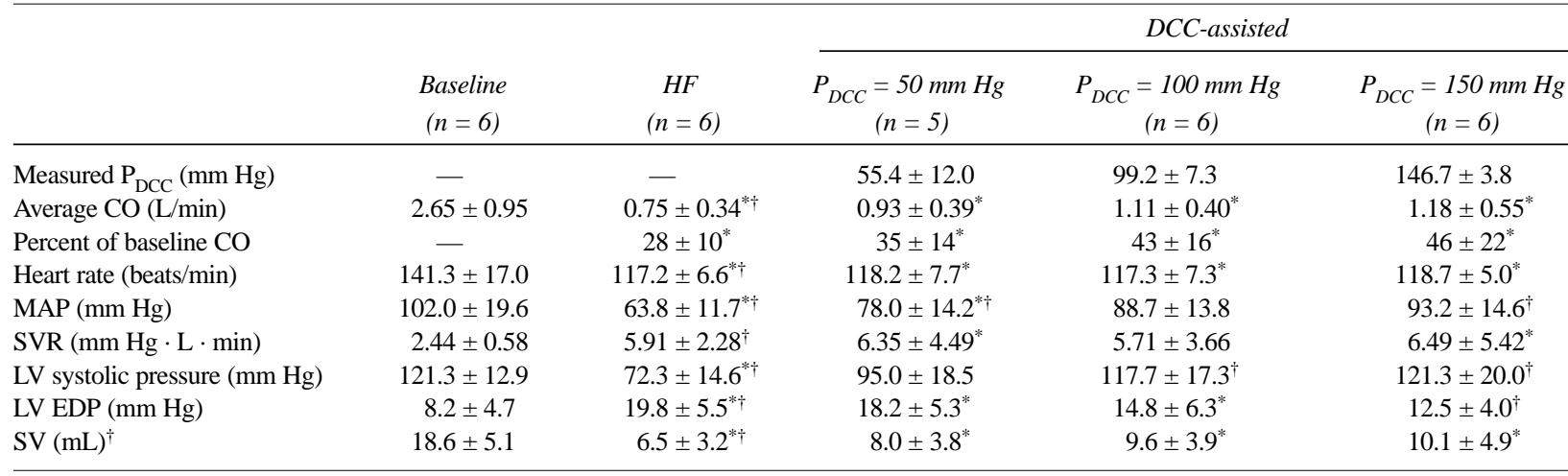

$\mathrm{P}_{\mathrm{Dcc}}$, Cardiac compression pressure; $H F$, heart failure; $C O$, cardiac output; $M A P$, mean arterial pressure; $S V R$, systemic vascular resistance; $E D P$, end-diastolic pressure; $S V$, stroke volume. All values are mean $\pm \mathrm{SD}$.

${ }^{*} P<.05$ versus baseline.

${ }^{\dagger} P<.05$ compared with value in column to the left from analysis based on RM-ANOVA with indicator variables.

Effect of volume loading and afterload reduction. To test whether cardiac output during DCC could be augmented through adjustments of ventricular load, we performed experiments in 6 animals in which normal saline solution and nitroprusside were infused. The results (Fig 4) show that as in the previous group of animals, embolization substantially decreased cardiac output, mean arterial pressure, LV systolic pressure, and stroke volume, whereas LV EDP and systemic vas- cular resistance increased compared with normal. Initiation of DCC at $132 \pm 24 \mathrm{~mm} \mathrm{Hg}$ induced a decrease in EDP of about $8 \mathrm{~mm} \mathrm{Hg}$ (from $20.6 \pm 2.2$ $\mathrm{mm} \mathrm{Hg}$ to $11.4 \pm 2.6 \mathrm{~mm} \mathrm{Hg}, P=.001)$, and cardiac output increased from $0.8 \pm 0.2$ to $1.4 \pm 0.5 \mathrm{~L} / \mathrm{min}(P=$ $.03)$, attaining a value of about $50 \%$ of normal (+DCC). When EDP was restored to $19.6 \pm 2.2 \mathrm{~mm} \mathrm{Hg}$ by saline infusion, cardiac output increased to $1.9 \pm 0.5 \mathrm{~L} / \mathrm{min}$ (+个P, $P=.04)$. Cardiac output was further increased to 

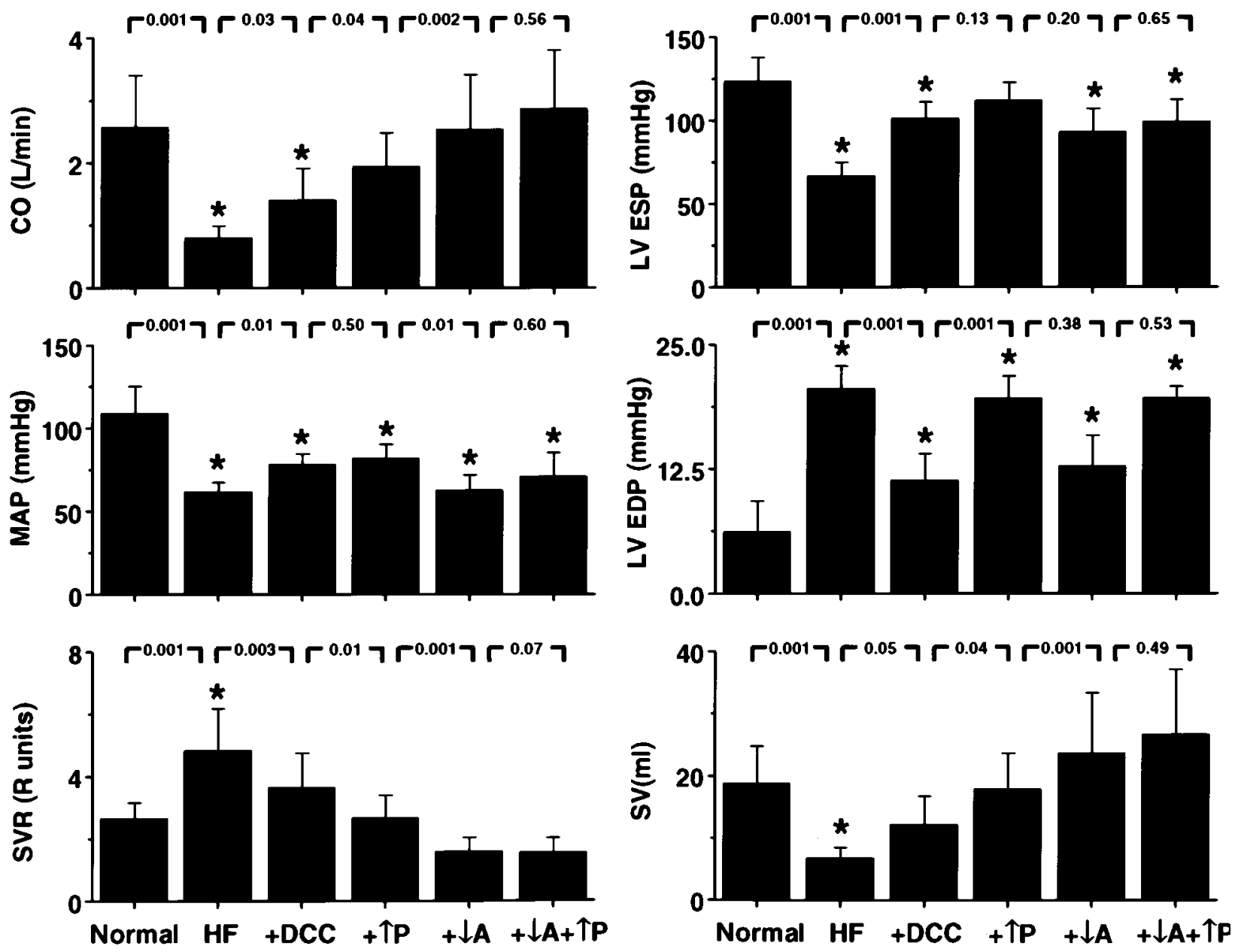

Fig 4. Summary of the effect of increasing preload $(\uparrow P)$ and reducing afterload $(\downarrow A)$ on DCC-assisted ventricular function. The cardiac output $(C O)$, mean arterial pressure $(M A P)$, systemic vascular resistance (SVR), LV end-systolic pressure ( $L V E S P)$, LV end-diastolic pressure ( $L V E D P)$, and stroke volume $(S V)$ are illustrated after each intervention. The heart rate varied slightly between the treatment conditions with respective values of (from left to right) $137.8 \pm 14.2,118.0 \pm 7.3,114.8 \pm 5.8,110.4 \pm 9.0,109.6 \pm 10.1,107.0 \pm 9.6$ beats $/$ min. ${ }^{*} P<.05$ versus baseline; $P$ values over each bar show the results of RM-ANOVA with indicator variables to parameterize specific contrasts.

a value of $2.5 \pm 0.9 \mathrm{~L} / \mathrm{min}(98 \%$ of normal) following the afterload reduction by nitroprusside $(+\downarrow \mathrm{A}, P=$ .002). However, the vasodilatory effect of nitroprusside was not restricted to arterial resistance but also caused venodilation, as evidenced by a (statistically not significant) drop in LV EDP from $19.6 \pm 2.2$ to $12.8 \pm 3.0$ $\mathrm{mm} \mathrm{Hg}(P=.38)$. LV EDP was again increased by another infusion of normal saline solution to a value of $19.8 \pm 1.3 \mathrm{~mm} \mathrm{Hg}$, at which point cardiac output increased to $2.8 \pm 1.1 \mathrm{~L} / \mathrm{min}$, or $105 \%$ of normal $(+\downarrow A+\uparrow P)$. Thus, active manipulation of preload and afterload provides an effective means of modulating the hemodynamic impact of DCC in vivo.

\section{Discussion}

Direct epicardial compression in synchrony with native ventricular contraction can increase effective contractility and overall pressure and flow-generating capacity of the heart (Figs 2 and 3). One effect of the acutely boosted ventricular pumping capacity is that filling pressures and volumes are decreased substantially on initiation of assistance, a factor that limits the immediate augmentation of cardiac output and systolic pressure. These observations are consistent with those of previous studies done in the setting of acute heart failure showing that, on average, cardiac output reached about $60 \%$ of normal during DCC. ${ }^{7}$ Cardiac 


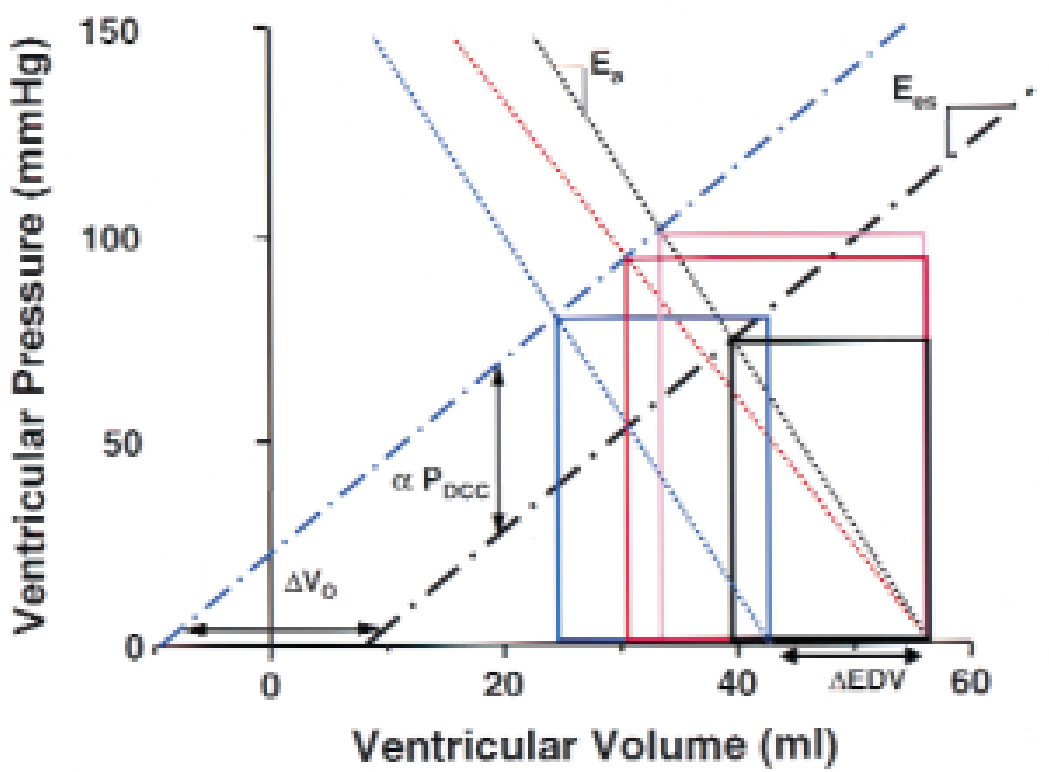

Fig 5. Current theories of ventricular-vascular coupling can be used to relate ventricular properties with those of the peripheral vascular system during DCC. Ventricular contractile state is quantified by the linear ESPVR where ventricular elastance $\left(E_{e s}\right)$ is the slope of the line and the unstressed ventricular volume $\left(V_{o}\right)$ is the volume axisintercept (solid lines). Afterload is quantified by the arterial elastance $\left(E_{a}\right)$ line where negative values for arterial elastance $\left(-E_{a}\right)$ is the slope of the line and the EDV is the volume-axis intercept (dotted lines). Arterial elastance $\left(E_{a}\right)$ is proportional to total peripheral resistance (TPR) and inversely proportional to the cardiac cycle duration (T): $\mathrm{E}_{\mathrm{a}} \propto \mathrm{TPR} / \mathrm{T}$. The intersection between the $\mathrm{E}_{\mathrm{a}}$ line and the ESPVR provides an estimate of the end-systolic pressurevolume point for the specified ventricular elastance $\left(\mathrm{E}_{\mathrm{es}}\right)$, ventricular volume $\left(\mathrm{V}_{\mathrm{o}}\right)$, end-diastolic volume (EDV), and arterial elastance $\left(\mathrm{E}_{\mathrm{a}}\right)$. Baseline heart failure conditions are shown in black; effects of DCC are in blue; active pre-loading during DCC is shown in pink; reduced afterload during DCC with volume loading is shown in red. Further details are provided in the text.

output and pressure augmentations are readily enhanced, however, by active adjustment of filling pressure through administration of intravenous fluids. Additional increases in cardiac output can be attained by counteracting arterial vasoconstriction normally encountered in heart failure, provided there is adequate blood pressure reserve during these interventions. In the present study, for hearts pumping only about $30 \%$ of normal cardiac output, DCC in combination with such preload and afterload manipulations could restore cardiac output to normal.

The interactions between the left ventricle, a DCC device, and the vascular load can be explained with the use of graphic representations of theories of ventricular vascular coupling ${ }^{14}$ (Fig 5). ESPVRs (dot-dashed lines), pressure-volume loops (solid lines), and effective arterial elastance lines $\left(E_{a}\right.$; dotted lines, an index of arterial afterload resistance) are shown in this figure. The baseline ESPVR during heart failure, accompanying pressure-volume loop, and baseline arterial elastance line are all shown in black. The influence of DCC is to shift the
ESPVR upward in an approximately parallel manner (blue dot-dashed line) by an amount proportional to $\mathrm{P}_{\text {DCC }}{ }^{6,7}$ When DCC is applied, preload is reduced, and the resulting pressure-volume loop, assuming no change in arterial properties, is shown by the solid blue loop. Stroke volume and mean arterial pressure (the width and height, respectively, of the pressure-volume loop) are only marginally increased over baseline. When preload is restored (pink pressure-volume loop), there are marked increases in both stroke volume and ESP. With reduction of arterial resistance (red dotted arterial elastance line), stroke volume is increased further at the expense of a slight reduction in ESP (red pressure-volume loop). Therefore, the resultant ventricular stroke volume and ESP are critically dependent on the interactions between the transmitted device compression pressure $\left(\alpha \mathrm{P}_{\mathrm{DCC}}\right)$, preload (EDP), afterload $\left(\mathrm{E}_{\mathrm{a}}\right)$, and the underlying ventricular function $\left(\mathrm{E}_{\mathrm{es}}\right)$.

For DCC devices to be used effectively in the setting of clinical heart failure, treatment algorithms will need to consider the underlying hemodynamic status of the 
patient and recognize the interrelations between $\mathrm{P}_{\mathrm{DCC}}$, preload, and afterload. Implementation of strategies to maximize hemodynamic effectiveness of DCC requires nothing more than hemodynamic monitoring and therapeutic interventions routinely used in the care of critically ill patients with heart failure. Peripheral arterial blood pressure monitoring and right heart catheterization (for assessing cardiac output and left and right heart filling pressures) provide all necessary information for guiding therapy. Fluid administration (such as normal saline or colloidal solutions) and diuretics provide the means for adjusting preload. Short-acting arterial dilators such as nitroprusside or some calcium channel blockers could be used for adjusting afterload resistance.

There are clear limits to the degree to which volume loading and afterload reduction can be used. Pulmonary capillary pressures need to be below the critical value necessary for normal gas exchange (typically $\leq 20 \mathrm{~mm} \mathrm{Hg}$ ) and arterial pressure needs to be high enough to maintain organ perfusion. Additionally, effectiveness of the device may vary depending on the degree of baseline ventricular dilation. ${ }^{4}$ Experiments performed thus far have used structurally normal hearts in which acute heart failure has been induced. However, it is predicted that the device will be significantly more effective in the setting of long-standing heart failure in which a significant amount of remodeling and dilation have occurred. Accordingly, much less load manipulation may be necessary in these patients. Thus, full understanding of the interactions between heart, device, and vascular load will ensure optimal use of the device in the clinical setting.

\section{REFERENCES}

1. Anstadt GL, Blakemore WS, Baue AE. A new instrument for prolonged mechanical massage (abstract). Circulation 1965; 31(Suppl):II-43.

2. Anstadt MP, Bartlett RL, Malone JP, Brown GR, Martin S, Nolan
DJ, et al. Direct mechanical ventricular actuation for cardiac arrest in humans: a clinical feasibility trial. Chest 1991;100:8692.

3. Kawaguchi O, Goto Y, Futaki S, Ohgoshi Y, Yaku H, Suga H. Mechanical enhancement and myocardial oxygen saving by synchronized dynamic left ventricular compression. J Thorac Cardiovasc Surg 1992;103:573-81.

4. Kawaguchi O, Goto Y, Futaki S, Ohgoshi Y, Yaku H, Suga H. The effects of dynamic cardiac compression on ventricular mechanics and energetics: role of ventricular size and contractility. J Thorac Cardiovasc Surg 1994;107:850-9.

5. Kawaguchi O, Goto Y, Ohgoshi Y, Yaku H, Murase M, Suga H. Dynamic cardiac compression improves contractile efficiency of the heart. J Thorac Cardiovasc Surg 1997;113:923-31.

6. Artrip JH, Wang J, Leventhal AR, Tsitlik JE, Levin HR, Burkhoff D. Hemodynamic effects of direct bi-ventricular compression studied in isovolumic and ejecting isolated canine hearts. Circulation 1999;99:2177-84.

7. Artrip JH, Yi GH, Levin HR, Burkhoff D, Wang J. The physiologic and hemodynamic evaluation of non-uniform direct cardiac compression. Circulation 1999,100(Suppl):II-236-43.

8. Suga $\mathrm{H}$, Sagawa K. Instantaneous pressure-volume relationships and their ratio in the excised, supported canine left ventricle. Circ Res 1974;35:117-26.

9. Burkhoff D, Alexander J Jr, Schipke J. Assessment of windkessel as a model of aortic input impedance. Am J Physiol 1988;255:H742-53.

10. Tsitlik JE, Halperin HR, Popel AS, Shoukas AA, Yin FCP, Westerhof N. Modeling the circulation with three-terminal electrical networks containing special nonlinear capacitors. Ann Biomed Eng 1992;20:595-616.

11. Todaka K, Leibowitz D, Homma S, Fisher PE, Derosa C, Stennett $\mathrm{R}$, et al. Characterizing ventricular mechanics and energetics following repeated coronary microembolization. Am J Physiol 1997;272:H186-94.

12. Knecht M, Burkhoff D, Yi GH, Popilskis S, Homma S, Packer M, et al. Coronary endothelial dysfunction precedes heart failure and reduction of coronary reserve in awake dogs. J Mol Cell Cardiol 1997;29:217-27.

13. Neter J, Wasserman W. Applied linear statistical models. Homewood (IL): Richard D. Irwin, Inc; 1974. p. 632-3.

14. Sunagawa K, Maughan L, Burkhoff D, Sagawa K. Left ventricular interaction with arterial load studied in isolated canine ventricle. Am J Physiol 1983;245:H773-80. 